\title{
Polarized Photometric Detector for High-Performance Liquid Chromatography
}

\author{
Atsushi Yamamoto*, Akinobu Matsunaga*, Kazuichi Hayakawa**, Eiichi Mizukami* and \\ Motoichi MrYaZAKI** \\ *Toyama Institute of Health, Nakataikoyama, Kosugi-machi, Toyama 939-03, Japan \\ **Faculty of Pharmaceutical Sciences, Kanazawa University, Takara-machi, Kanazawa 920, Japan
}

\begin{abstract}
A photometric detector for an optically active compound is described in which two polarizers are set on both sides of the flow cell. When a nonchromophoric, optically active compound passes through the cell, the intensity of natural light transmitted through the polarizers and flow cell is changed. The detection of such a sample is possible photometrically. A large inclination angle between the polarizers increases the absorbance change and allows dextrorotatory and levorotatory to be differentiated. The direct HPLC/photometric detection of sugars is presented as an example of this device.
\end{abstract}

Keywords Polarized photometric detector, high-performance liquid chromatography, polarizer, optically active compound, sugar

An optical-rotation detection method utilizing photometers has been developed to detect nonchromophoric, optically active compounds. When such a compound is placed between two polarizers, the intensity of the light transmitted through the compound and polarizers is changed. By using this phenomenon, the detection of an optically active compound is possible utilizing a conventional photometric detector in which two polarizers are set on both sides of the flow cell. We call this new selective photometric monitoring method "polarized photometric detection (PPD)".

When a previous type photometric absorbance detector was used for the determination of sugar, derivatization was necessary ${ }^{1}$, since sugar has no convenient UV absorption bands. Its chirality, on the other hand, leads to optical activity. Several attempts to utilize an opticalrotation detector based on the Faraday effect have been reported. $^{2-4}$ In this paper, therefore, we describe the principle of PPD using sugars as examples.

\section{Theoretical}

We first define a new function, $f(x)$, as follows: $f(x)$ has a maximum at $x= \pm n \pi(n=0,1,2, \cdots)$ and converges to zero at $x= \pm n \pi / 2$. If the inclination of the plane of polarization of the artificial polarizer relative to the incident planar polarized light is $x$, the intensity of light transmitted through the polarizer obeys the function $f(x)$. The intensity of natural, unpolarized light $\left(I_{0}\right)$ transmitted through the two polarizers in series is given by

$$
I_{0}=\int_{-\pi / 2}^{\pi / 2}\{f(x)\}^{2} \mathrm{~d} x .
$$

When an optically active sample is placed between the two polarizers, the plane of polarization of the first polarizer is rotated by an angle $\alpha$. The intensity of this light $(I)$ transmitted through the second polarizer is given by

$$
I=\int_{-\pi / 2}^{\pi / 2} f(x) \cdot f(x+\alpha) \mathrm{d} x .
$$

This equation can be rewritten using the Taylor's series as

$$
\begin{aligned}
& I=I_{0}+\alpha \int_{-\pi / 2}^{\pi / 2} f(x) \cdot f^{\prime}(x) \mathrm{d} x+ \\
& \left(\alpha^{2} / 2\right) \int_{-\pi / 2}^{\pi / 2} f(x) \cdot f^{(2)}(x) \mathrm{d} x+\cdots+ \\
& \quad\left(\alpha^{n} / n !\right) \int_{-\pi / 2}^{\pi / 2} f(x) \cdot f^{(n)}(x) \mathrm{d} x+\cdots \\
& \text { Since } \int_{-\pi / 2}^{\pi / 2} f(x) \cdot f^{(2 n+1)}(x) \mathrm{d} x \text { is zero, replacing } \int_{-\pi / 2}^{\pi / 2} f(x) \cdot \\
& f^{(2 n)}(x) \mathrm{d} x \text { with constant } C_{n} \text { gives } \\
& I=I_{0}+\left(C_{1} / 2\right) \alpha^{2}+\cdots+\left(C_{n} /(2 n) !\right) \alpha^{2 n}+\cdots
\end{aligned}
$$

The absorbance (Abs) of the sample can be expressed as 


$$
\text { Abs }=\log I_{0}-\log I .
$$

If we let $I_{0}$ be unity and expand this equation in series, while ignoring the smaller factors in each term, we obtain

$$
\begin{aligned}
\mathrm{Abs} \propto & \left(C_{1} / 2\right) \alpha^{2}+\left(C_{2} / 24-C_{\mathrm{I}}{ }^{2} / 8\right) \alpha^{4}+\cdots \\
& +\left(-C_{1} / 2\right)^{n-2}\left(C_{2} / 24-C_{1^{2}} / 4 n\right) \alpha^{2 n}+\cdots .
\end{aligned}
$$

Since the relation $I_{0} \simeq-C_{1}<C_{2}$ is generally found to hold for $f(x)$, the coefficients from $\alpha^{4}$ onwards become sufficiently small for the terms to be negligible and the following equation is derived from $\mathrm{Abs}$ over a wide range of $\alpha$ :

$$
\mathrm{Abs}=C_{0} \alpha^{2} \quad\left(C_{0}=\text { constant }\right) .
$$

Equation (1) implies that the square of the sugar concentration is proportional to the absorbance. Consequently, in order to increase the detection sensitivity, the second polarizer should be tilted significantly from the first. In this case, let the inclination angle between the two polarizers be $R$ and the optical rotation of a sample at concentration $C$ be $\alpha_{c}$; then, the change in the absorbance ( $\Delta \mathrm{Abs})$ can be expressed as

$$
\Delta \mathrm{Abs} \propto\left(\alpha_{c}+R\right)^{2}-R^{2}=\alpha_{c}\left(\alpha_{c}+2 R\right) .
$$

Since $\alpha_{c} \ll R$, the above equation can be rewritten as

$\Delta \mathrm{Abs} \propto \boldsymbol{R} \boldsymbol{\alpha}_{c}$.

Equation (2) implies that $\Delta \mathrm{Abs}$ is directly proportional to the sugar concentration, and that its slope depends on the value $R$.

\section{Experimental}

The HPLC system included a Shimadzu (Kyoto, Japan) LC-5A pump, a Rheodyne (Cotati, CA, USA) 7125 injector (100 $\mu$ l loop) and a Shimadzu SPD-6AV UV-visible detector. A block diagram of the detector is shown in Fig. 1. The polarizers used were Polaroid (Norwood, MA, USA) types HN32 for the visible region and HNP'B for the visible and near-ultraviolet regions; those were placed on both sides of the flow cell in the above detector.

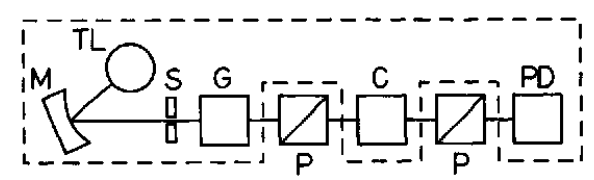

Fig. 1 Block diagram of the polarized photometric detector: TL indicates the tungsten lamp; $M$, the mirror; $S$, the slit; $G$, the grating; $P$, the polarizer; $C$, the cell; and $P D$, the photodiode. The part within the dashed line represents a commercially produced photometric detector.
Solutions of $\mathrm{D}$-fructose $\left([\alpha]_{\mathrm{D}}^{20}=-92^{\circ}\right)$, D-glucose $\left([\alpha]_{\mathrm{D}}^{20}=+53^{\circ}\right)$, sucrose $\left([\alpha]_{\mathrm{D}}^{20}=+66^{\circ}\right)$, and maltose $\left([\alpha]_{\mathrm{D}}^{20}=+130^{\circ}\right)$ were prepared in distilled water.

The chromatographic separation of the sugars was performed on a $25 \mathrm{~cm} \times 4.6 \mathrm{~mm}$ i.d. amide bonded column (Tosoh, Tokyo, Japan, TSK gel Amide 80) maintained at $70^{\circ} \mathrm{C}$. An $80 \%$ ethanol eluent was delivered at $0.6 \mathrm{ml} / \mathrm{min}$.

\section{Results and Discussion}

Figure 2 shows the spectral energy distribution in an SPD-6AV detector equipped with polarizers. The ordinate axis represents the relative intensity of energy, that is the intensity of light transmitted through the polarizers, multiplied by the product of tungsten lamp spectroscopic characteristics, grating characteristics and photodiode response. Since the sugars show plain optical rotatory dispersion curves, their optical rotation in terms of the absolute value increases in the ultraviolet region. ${ }^{5}$ However, as shown in Fig. 2, the relative intensity of energy through the polarizers decreases as the measuring wavelength becomes shorter. This results in an increase in the base line noise. Consequently, we found that the best sensitivity for sugar detection in this system was attained at a wavelength of around $490 \mathrm{~nm}$.

The relationship between the angle made by the two polarizers and the absorbance at $490 \mathrm{~nm}$ conforms to Eq. (1) when $\alpha$ is less than 1 radian for both polarizers.

On the basis of the above findings, chromatograms of sugars were obtained at $490 \mathrm{~nm}$ by turning the second polarizer significantly clockwise. Figure 3 shows a typical separation of sugars using the normal-phase column mentioned previously, with the inclination angle between the two polarizers set at 0.937 radian. Although the intensities of the sugar peaks increased in proportion to the degree of rotation of the second polarizer, the base line noise also increased as the quantity of light decreased. The inverse peak of fructose in Fig. 3 is due

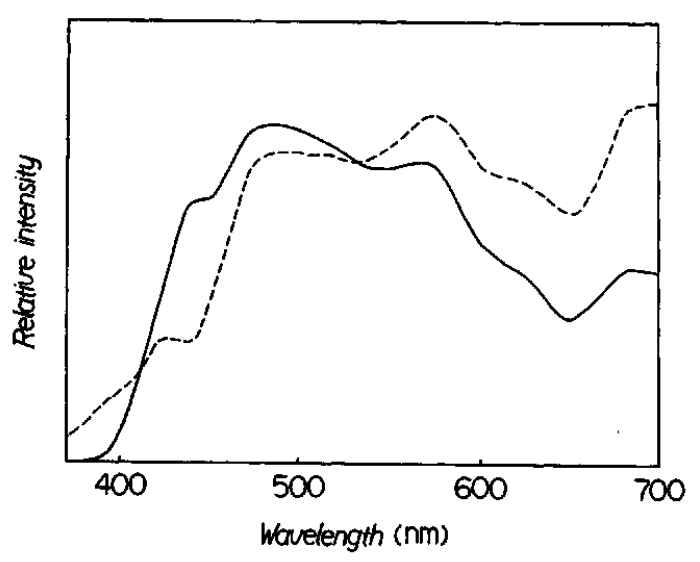

Fig. 2 Spectral energy distribution through the polarizers set in the SPD-6AV detector. _-; HN32, --.-; HNP'B. 


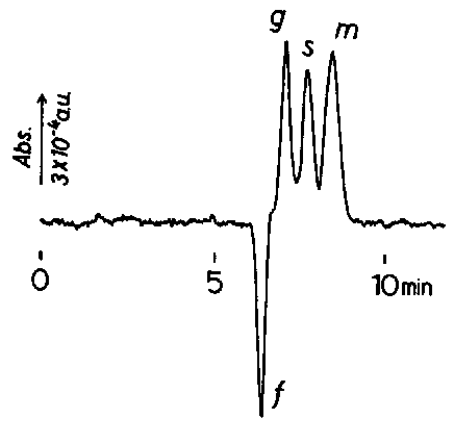

Fig. 3 Chromatogram of sugar standards. The chromatographic conditions are given in the text. Peaks: $\mathrm{f}$, fructose $(3.3 \mu \mathrm{mol}) ; \mathrm{g}$, glucose $(2.8 \mu \mathrm{mol}) ; \mathrm{s}$, sucrose $(0.9 \mu \mathrm{mol}) ; \mathrm{m}$, maltose $(1.1 \mu \mathrm{mol})$.

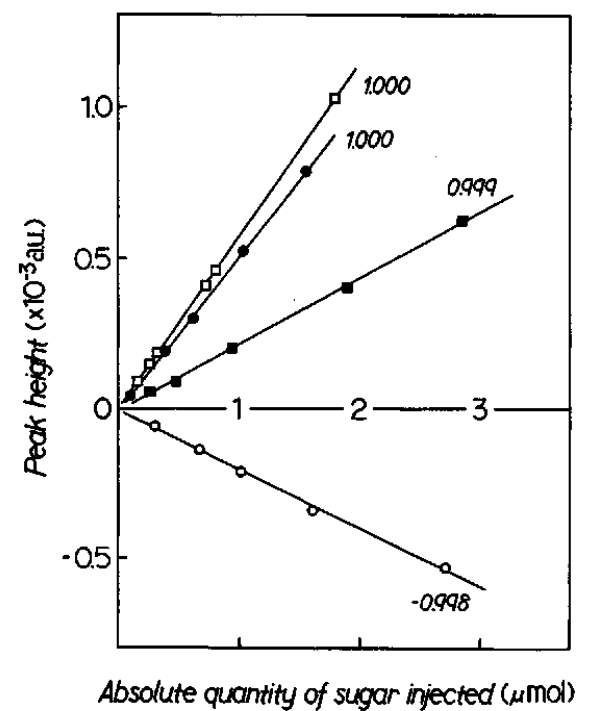

Fig. 4 Calibration curves of several sugars. The chromatographic conditions are the same as those in Fig. 3. Symbols: $\square$, sucrose; $\bigcirc$, maltose; $\square$, glucose; $\bigcirc$, fructose. The numbers at the end of the lines are the coefficients of the correlation. to it being levorotatory. Figure 4 shows the calibration curves of sugars under the same conditions as in Fig. 3. They had good linearities over these concentration ranges in accordance with Eq. (2) and their detection limits were all below $0.1 \mu \mathrm{mol}$. The standard deviations for repeated injections of the mixture in Fig. 3 were less than $2 \%$ for each sugar.

It is known that reducing sugars generally causes mutarotation. The fact that no change in the peak heights was observed as time proceeded suggests immediate isomerization within the column.

In conclusion, PPD is useful in the general HPLC detection of not only sugars, but also other optically active compounds. The sensitivity can be further optimized with a careful selection of light sources and/or polarizers.

We thank M. Nishimura of Shimadzu Corporation, Kyoto Analytical Applications Center for technical support.

\section{References}

1. K. Shinomiya, H. Toyoda, A. Akahoshi, H. Ochiai and T. Imanari, J. Chromatogr., 387, 481 (1987).

2. E. S. Yeung, L. E. Steenhoek, S. D. Woodruff and J. C. Kuo, Anal. Chem., 52, 1399 (1980).

3. J. C. Kuo and E. S. Yeung, J. Chromatogr., 223, 321 (1981).

4. D. K. Lloyd, D. M. Goodall and H. Scrivener, Anal. Chem., 61, 1238 (1989).

5. T. L. Harris, E. L. Hirst and C. E. Wood, J. Chem. Soc., $1937,848$.

(Received July 1, 1991)

(Accepted August 12, 1991) 